\title{
A Systematic Review and Meta-analysis of the Efficacy of Intraoperative Vascular Doppler Ultrasonography for Vein Ligations and Artery Preservations in Microsurgical Subinguinal Varicocelectomy
}

\author{
Muhammad Husni Tamrina, ${ }^{\mathrm{a}, \mathrm{b}}$ Doddy Moesbadianto Soebadi ${ }^{\mathrm{a}, \mathrm{b}}$, Mohammad \\ Ayodhia Soebadi ${ }^{\mathrm{a}, \mathrm{c}}$, Yudhistira Pradnyan Klopinga,b Furqan Hidayatullah ${ }^{\mathrm{a}, \mathrm{b}}$, \\ Fikri Rizaldia,b \\ ${ }^{\mathrm{a}}$ Department of Urology, Faculty of Medicine, Universitas Airlangga, Surabaya, East Java, Indonesia \\ ${ }^{\mathrm{b}}$ Dr. Soetomo General-Academic Hospital, Surabaya, East Java, Indonesia \\ ${ }^{\mathrm{c}}$ Rumah Sakit Universitas Airlangga Teaching Hospital, Surabaya, East Java, Indonesia
}

\begin{abstract}
The gold standard of varicocele management is microsurgical subinguinal varicocelectomy. This procedure leads to better preservation of the testicular artery and lymphatic vessels. However, sometimes several vessels were in complex position causing the dissection to be more difficult. By using IVDU, more arteries and veins could be identified to acquire better sperm outcomes. It's uncertain if the Doppler Ultrasonography has a role in varicocele therapy. The goal of this systematic review and meta-analysis was to see how effective microsurgical subinguinal varicocelectomy with intraoperative vascular Doppler ultrasonography compared to without intraoperative vascular Doppler ultrasonography. Randomized Controlled Trials (RCTs), non- randomized trials, and comparative observational studies were searched and screened in the Embase, Medline, and Scopus databases for research examining the role of intraoperative Doppler ultrasonography. The Cochrane risk of bias (RoB) tool 2 was used to assess RCT bias, whereas the ROBINS-I tool was utilized to assess non-randomized trial bias. The New Ottawa Scale was used to evaluate observational research. The systematic search and screening yielded five suitable studies, including three RCTs, one non-randomized trial, and one observational research. Between patients receiving IVDU and those having the treatment without IVDU, there is a significant difference in the number of ligated veins (MD 0,94; 95 percent CI 0,62-1,26; p0,00001) and preserved arteries (MD 0,43; 95 percent CI 0,30-057; p0,00001). Patients receiving IVDU had better ligated veins and preserved arteries than patients having the surgery without IVDU.
\end{abstract}

Keywords: Varicocele, Microsurgical subinguinal varicocelectomy; Intraoperative Vascular Doppler Ultrasonography 


\section{Introduction}

The enlargement of pampiniform plexus from spermatic vein along spermatic cord was defined as varicocele. [1]. Varicocele was strongly associated with infertility in men. The prevalence of varicocele was $15 \%$ from normal male population, $25 \%$ in male with abnormal sperm analysis, and $30-40 \%$ in male with infertility. The pathological process of varicocele was the dilataion of internal spermatic vein which blood refluxes and returns to pampiniform plexus [2].

There are several types of varicele treatments. Varicocele treatment exists in a variety of forms. Between spermatic vein ligation, antegrade or retrograde sclerotherapy, microsurgical varicocelectomy, and laparoscopic varicocelectomy, the best therapeutic option is still up for debate. Treatment for varicocele was indicated by infertility and chronic discomfort. As a result, varicocelectomy can raise natural pregnancy rates by 2.87 times in couples when the male has a poor sperm parameter and a palpated varicocele [3]. In comparison to previous varicocele treatments, microsurgical varicocelectomy has been the gold standard in lowering complication and recurring varicocele. Microsurgical varicocelectomy can be performed inguinally or subinguinally with the use of a microscope. This will decrease testicular artery ligation, which causes roughly $1 \%$ testicular atrophy [4]. When compared to inguinal microsurgical varicocelectomy, subgingiinal microsurgical varicocelectomy makes it easier to identify the spermatic artery and vein. Because this procedure had risk for arterial damage, Intraoperative Vascular Doppler Ultrasonography may be helpful in identifying the artery. Intraoperative Vascular Doppler Ultrasonography might be used to help detect the spermatic vein and artery in the spermatic cord during microsurgical varicocelectomy. Vascular Doppler Ultrasonography is predicted to lessen the complication of testicular atrophy caused by testicular artery damage during microsurgery sessions by increasing the number of recognized and preserved arteries. The existence of recurrent venous blood flow reflux, which causes recurrent varicocele, was strongly associated with the number of ligated spermatic veins, which was positively correlated with total concentration and sperm motility [5].

Intraoperative Vascular Doppler Ultrasonography in Subinguinal Microsurgical Varicocelectomy was found to shorten operative time, increasing the number of artery preservation and identification, and increasing the number of ligated veins in previous research [6]. As a result, the goal of this systematic review and metaanalysis is to evaluate more about the differences between microsurgical subinguinal varicocelectomy with and without Intraoperative Vascular Doppler Ultrasonography.

\section{Methods}

\section{Search Strategy and Eligbility Criteria}

A systematic search was conducted in numerous databases, including PubMed, EMBASE, Scopus, and Science Direct, according to the predetermined Patient, Intervention, Comparison, and Outcome criteria (PICO). The time limitation of the research examined in this analysis was not restricted. The goal of this search strategy was to discover studies that compared the use of Intraoperative Vascular Doppler Ultrasonography without the use of Intraoperative Vascular Doppler Ultrasonography in varicocele patients who received subinguinal microsurgical varicocelectomy. Studies were included if (1) they were in RCT, prospective or retrospective study, (2) the study had data comparing the effectiveness of subinguinal microsurgical varicocelectomy with and without Intraoperative Doppler Vascular Ultrasonography, (3) the patients were male and over the age of 18, and (4) they were published in a peer-reviewed journal. 
Quality Assessment of the Included Studies

Individual papers were evaluated for quality using the Cochrane Risk of Bias Tools for RCT studies, and prospective study and the Newcastle Ottawa Scale (NOS) for retrospective studies. In Cochrane Risk of Bias Tools, several domains of bias were assessed in this study including bias due to confounding, bias due to participant selection, bias in intervention classification, bias due to deviations from intended interventions, bias due to missing data, bias in outcome measurement, and bias in selection of reported result. Each domain was graded using two criteria: some concerns and low concerns. In Newcastle Ottawa Scale, if the score was more than 6 , the study was rated as good quality.

\section{Data Extraction}

Two writers worked independently to extract data by filling out an extraction data table. Year of publication, author's name, mean age, research design, intervention, and cross tabulation of desired result were all tabulated in the table. Any conflicts or inconsistencies were handled through conversation with the other writers. Study design and characteristics, baseline characteristics of study samples, intervention, outcome, and risk of bias were all retrieved.

\section{Statistical Analysis}

To compare factors in each trial, statistical analysis was performed using pooled analysis. The number of proportions and samples used in each study were evaluated using odds ratio (OR) with a 95 percent confidence interval (CI) in the form of dichotomous data. Each variable was considered statistically significant if the $\mathrm{P}$ value was less than 0.05 . In the case of continuous data, the analysis was performed using mean difference and standard deviation in each group. $\mathrm{I}^{2}$ test was used to determine the degree of heterogeneity between trials. If $\mathrm{I}^{2}>50 \%$, a high level of heterogeneity was considered, and a random-effect model was applied. If $\mathrm{I}^{2}$ was less than $50 \%$, a fixed effect model was utilized, and low heterogeneity was considered. Each analysis was presented in the form of forest plot and descriptive analysis. For this investigation, RevMan software version 5.4 for Windows was employed.

\section{Results}

Selection of Studies

The PRISMA procedure flowchart was used to conduct a systematic search in PUBMED, EMBASE, Scopus, and Science Direct, yielding 4201 articles (Figure 1). There were 1528 article duplications discovered in those databases, which were eventually eliminated. After title and abstract screening, 48 papers were selected for further screening, and their eligibility was assessed. Finally, the quantitative analysis of this study included 5 papers that met the inclusion criteria. 


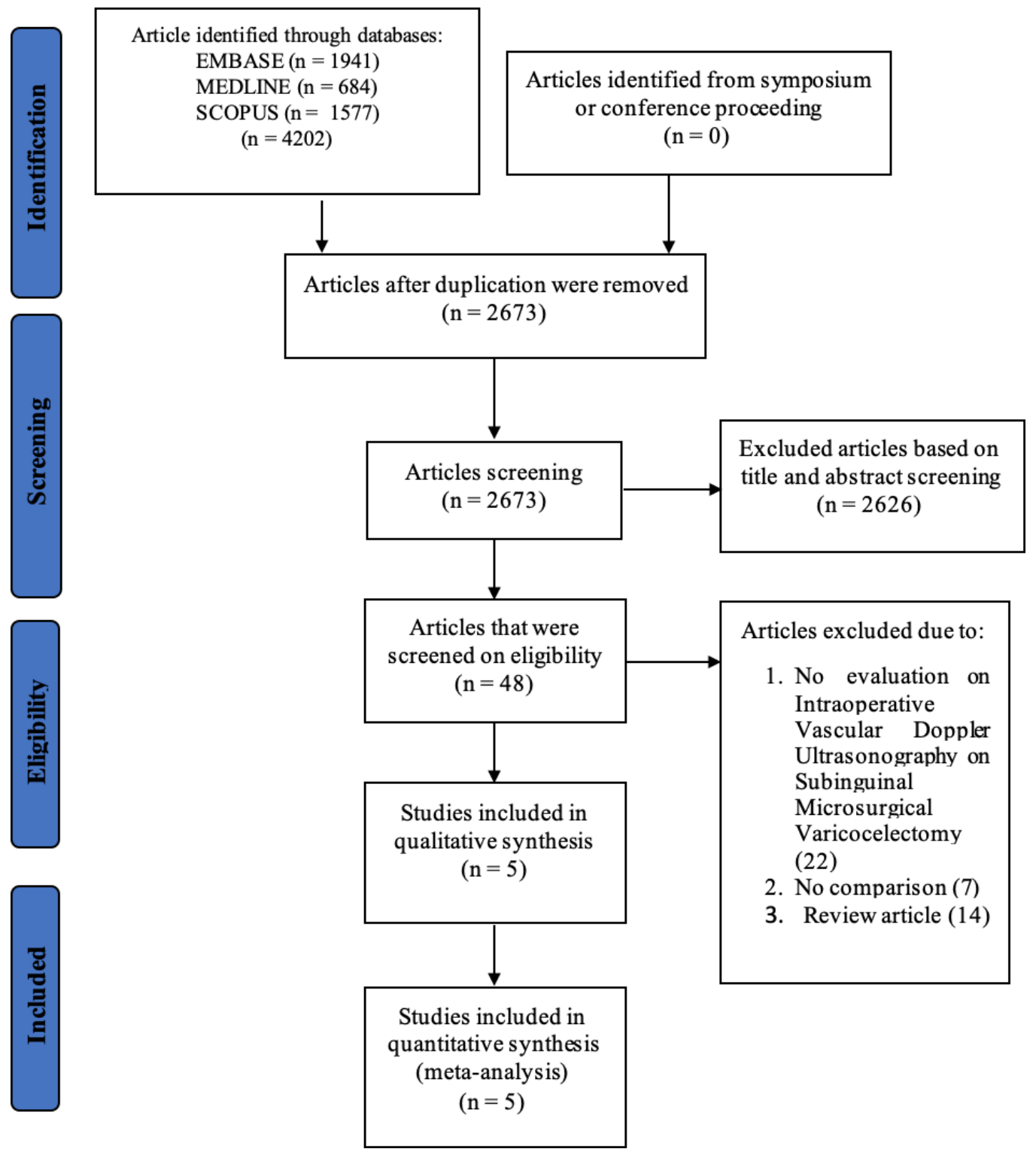

Figure 1. PRISMA flowchart in the systematic search

Baseline Characteristics of Included Studies

In our meta-analysis, we analyzed 5 papers [5-9] that compared the use of IVDU against no IVDU in subinguinal microsurgical varicocelectomy procedures. Three RCTs and two observational studies made up our individual investigations. Table 1 summarized the baseline characteristics of the included trials, which 
were broken down by intervention, location, and severity level. Our study's mean age was 32 years old. This research has a total of 758 participants.

\section{Risk of Bias Assessment}

The Newcastle Ottawa Scale (NOS) and the Cochrane Risk of Bias (RoB) questionnaire were used to assess the risk of bias. The Cochrane ROB tools were used to analyze RCTs and prospective study, whereas NOS was used to examine retrospective studies. The risk of bias in RCT studies was assessed (Figure 2A), and it was found that there was unclear bias in the area of D5 (bias in the selection of reported results) in Guo et al, 2015, and the D4 domain (bias in outcome measurement) in Lv et al, 2017. The rest of the domain had a low risk of bias. Selection bias, performance bias, and detection bias were the evaluations in risk of bias for three RCTs. In prospective research, the risk of bias evaluation revealed that all domains had a low risk of bias (Figure 2B). The NOS instrument was used to assess the likelihood of bias in a retrospective study, and a minimum score of 6 was found, which is considered a good quality score (Figure 2C).

(a)

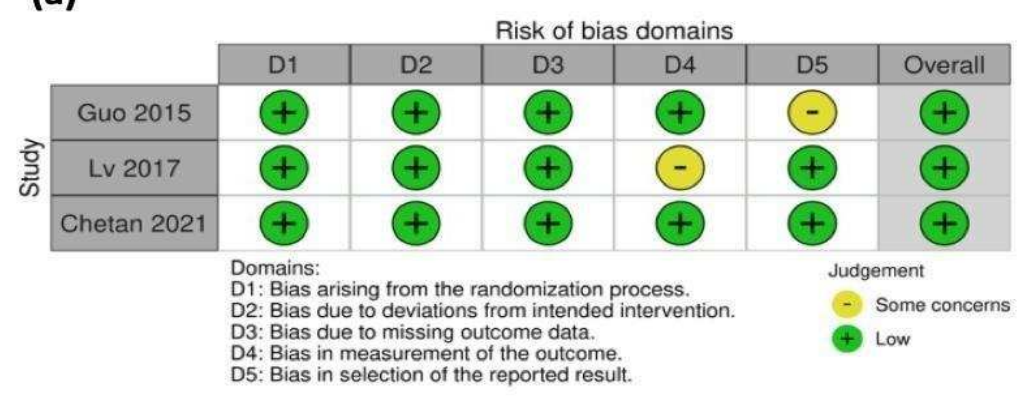

(b)

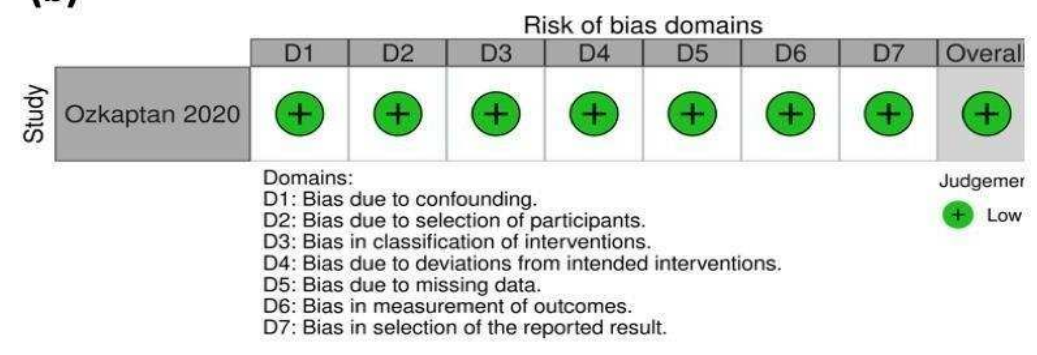

(c)

\begin{tabular}{|l|c|c|c|c|}
\hline \multirow{2}{*}{ Author (Year) } & \multicolumn{4}{|c|}{ Quality Score } \\
\cline { 2 - 5 } & Selection & Comparison & Exposure & Total \\
\hline Cocuzza (2010) & $* *$ & $*$ & $* * *$ & 6 \\
\hline
\end{tabular}

Figure 2. Risk of Bias Assessment (a) in RCT studies using Cochrane ROB Tool 2, (b) Prospective study using Cochrane ROB tool 2, and (c) Observational studies using Newcastle Ottawa Scale (NOS) 


\section{Ligated Veins}

The differential in number of ligated veins was reported in all of the studied studies [5-9]. The difference in mean number of ligated veins between the group of subinguinal microsurgical varicocelectomy with Intraoperative Vascular Doppler Ultrasonography compared to the group of subinguinal microsurgical varicocelectomy without Intraoperative Vascular Doppler Ultrasonography was statistically significant (MD 0.94; 95 percent CI $0.62-1.26$; $\mathrm{p}<0.00001$ ) as shown in Figure 3. Because of the low heterogeneity amongst the studies $\left(\mathrm{I}^{2}=6 \%\right)$, a fixed-effects model was utilized.

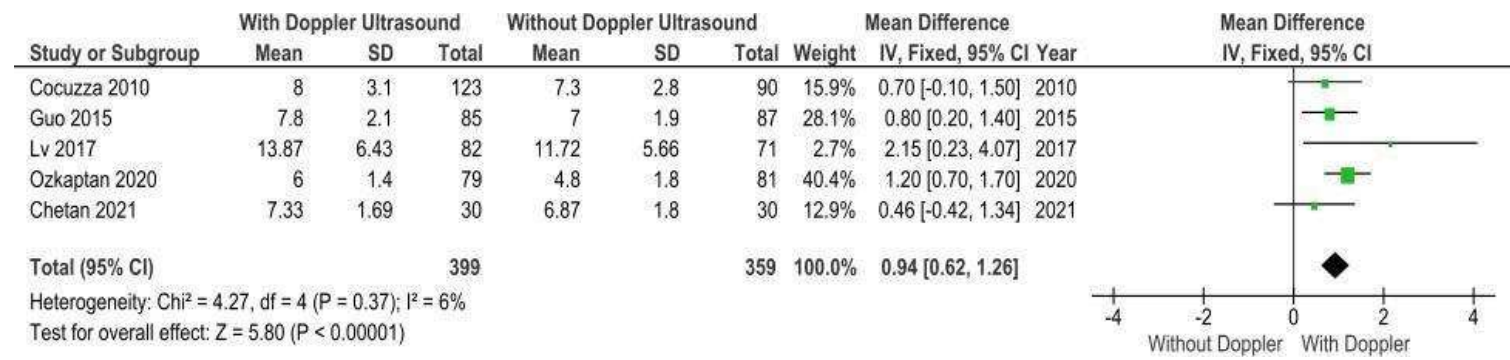

Figure 3. Forrest plot analysis of the difference in number of ligated veins between the use of IVDU and without the use of IVDU in subinguinal microsurgical varicocelectomy

\section{Preserved Arteries}

All of the studies that were included [5-9] in the analysis of the difference in the average number of preserved arteries. The mean number of preserved arteries between the groups of using Intraoperative Vascular Doppler Ultrasonography and not using Intraoperative Vascular Doppler Ultrasonography in subinguinal microsurgical varicocelectomy was statistically significant (MD 0.43; 95 percent CI $0.30-0.57$; p0,00001), according to the results of forest plot (Figure 4 ) analysis. Due to minimal heterogeneity between trials $\left(\mathrm{I}^{2}\right.$ test $\left.=55 \%, \mathrm{p}=0.07\right)$, a fixed-effects model was chosen.

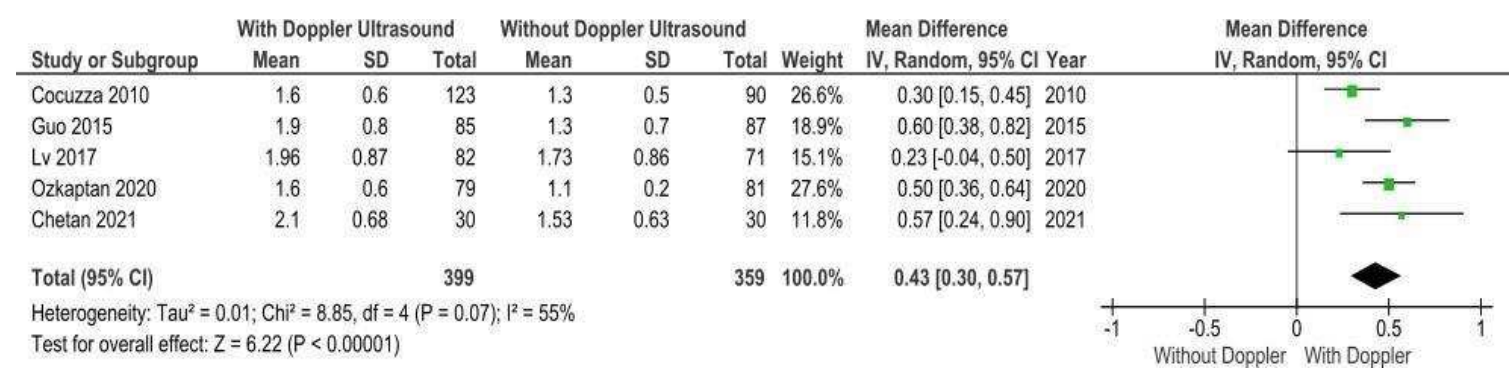

Figure 4. Forrest plot analysis of average number of preserved arteries in subinguinial microsurgical varicocelectomy 
Table 1. Baseline Characteristics of Included Studies

\begin{tabular}{|c|c|c|c|c|c|c|c|}
\hline \multirow[t]{2}{*}{ Study (year) } & \multirow{2}{*}{$\begin{array}{l}\text { Study } \\
\text { Design }\end{array}$} & \multicolumn{2}{|c|}{ Samples Number } & \multicolumn{2}{|c|}{ Mean age (years) } & \multirow[b]{2}{*}{ Microscope } & \multirow{2}{*}{$\begin{array}{c}\text { Vascular } \\
\text { Doppler } \\
\text { Ultrasonography }\end{array}$} \\
\hline & & $\begin{array}{l}\text { With Doppler } \\
\text { Ultrasonography }\end{array}$ & $\begin{array}{l}\text { Without } \\
\text { Doppler } \\
\text { Ultrasonography }\end{array}$ & $\begin{array}{c}\text { With } \\
\text { Doppler } \\
\text { Ultrasound }\end{array}$ & $\begin{array}{c}\text { Without } \\
\text { Doppler } \\
\text { Ultrasound }\end{array}$ & & \\
\hline (Guo et al., 2015) & RCT & 85 & 87 & $\begin{array}{c}30.45 \pm \\
5.67\end{array}$ & $\begin{array}{c}31.20 \pm \\
6.25\end{array}$ & $\begin{array}{c}\text { VM900 } \\
\text { (Moller- } \\
\text { Wedel) } \\
\text { (Zoom in 8- } \\
\text { 15x) } \\
\text { Leica }\end{array}$ & $\begin{array}{l}\text { VTI } 20 \mathrm{MHz} \\
\text { (Nashua, NH) }\end{array}$ \\
\hline (Lv et al., 2017) & RCT & 82 & 71 & $\begin{array}{c}28.16 \pm \\
8.20\end{array}$ & $\begin{array}{c}28.51 \pm \\
6.99\end{array}$ & $\begin{array}{l}\text { M520-MC- } \\
1 \\
(\text { Zoom in } \\
8-15 x)\end{array}$ & $\begin{array}{l}\text { VTI } 20 \mathrm{MHz} \\
\text { Microvascular } \\
\text { Doppler }\end{array}$ \\
\hline $\begin{array}{l}\text { (Chetan et al., } \\
\text { 2021) }\end{array}$ & $\mathrm{RCT}$ & 30 & 30 & $34.27 \pm 3.8$ & $\begin{array}{c}33.93 \pm \\
5.43\end{array}$ & NA & NA \\
\hline $\begin{array}{l}\text { (Cocuzza et al., } \\
2010)\end{array}$ & Prospective & 123 & 90 & $30.2 \pm 7.7$ & $29.3 \pm 7.7$ & $\begin{array}{c}\text { Zeiss } \\
(\text { Zoom 8- } \\
15 x)\end{array}$ & $\begin{array}{l}\text { VTI 9,3 MHz } \\
\text { (Nashua, NH) }\end{array}$ \\
\hline $\begin{array}{l}\text { (Özkaptan et al., } \\
\text { 2020) }\end{array}$ & Retrospective & 79 & 81 & $29.01 \pm 5.9$ & $\begin{array}{c}30.01 \pm \\
5.29\end{array}$ & $\begin{array}{c}\text { Leica M525 } \\
\text { (Zoom 8- } \\
15 x)\end{array}$ & $\begin{array}{c}\text { VTI } 20 \mathrm{MHz} \\
\text { Microvascular } \\
\text { Doppler System }\end{array}$ \\
\hline
\end{tabular}




\section{Discussion}

One of the standard treatments for varicocele was subinguinal microsurgical varicocelectomy. The capacity to visualize artery, vein, lymph, and nerve was one of the advantages of subinguinal microsurgical varicocelectomy. Marmar introduced subinguinal microsurgical varicocelectomy in 1985, and Goldstein refined it in 1992. In infertile and clinically palpated varicocele patients, microsurgical varicocelectomy has proven to be a successful therapy when compared to open microvaricocelectomy and laparoscopic varicocelectomy

Intraoperative Vascular Doppler Ultrasonography (IVDU) may help identify the spermatic vein and artery during subinguinal microsurgical varicocelectomy by detecting arterial pulsation in the sonography wave. Juho et al. and Gudeloglu et al. completed previous studies that supported the use of sonography in varicocelectomy. In each of these investigations, the use of Intraoperative Vascular Doppler Ultrasonography enhanced the number of ligated veins and intact arteries, lowering the rate of varicocele recurrence and testicular artery injury [1,10]. Vascular injury during microsurgical varicocelectomy might result in DNA fragmentation, which reduces sperm quality and decreases the proportion of sperm after surgery.

In this systematic review, all studies compared the number of ligated veins and preserved arteries, and a significant difference was found in the number of ligated veins (MD 0.94; 95 percent CI 0.62 - 1.26; p0.00001) and number of preserved arteries (MD 0.43; 95 percent CI $0.30-0.57$; p0.00001) between the IVDU and non-IVDU groups. This was linked to the advantage of subinguinal microvaricocelectomy, which allowed for the identification of more spermatic veins and arteries, resulting in a higher number of ligated and preserved arteries [11]. Through arterial pulsation, Intraoperative Vascular Doppler Ultrasonography could aid in the identification of vein and spermatic artery during subinguinal microsurgical varicocelectomy [3].

Overall, this study found that when patients had subinguinal microsurgical varicocelectomy with intraoperative vascular doppler ultrasonography, the number of ligated veins and preserved arteries increases. This finding suggests that IVDU may play an important role in subinguinal microsurgical varicocelectomy procedures. The use of IVDU could also improve sperm count in the early stages after surgery, according to clinical findings. This study solely looked at observed clinical evaluations; no mollecular mechanism that could explain the link between preserved arteries and increased number of ligated veins was investigated. However, according to prior research, improvements in vascular health may be linked to improvements in these clinical parameters.

This systematic review and meta-analysis had number of drawbacks. Because the number of studies included in this systematic review was still limited, the analysis' outcome was less than ideal. There were no studies that evaluated pregnancy rate after subinguinal microsurgical varicocelectomy with and without the use of IVDU. Furthermore, a long-term evaluation may be required to offer evidence of the patient's reproductive status, particularly in terms of conception ability.

\section{Conclusion}

The use of IVDU in subinguinal microsurgical varicocelectomy has the advantages of a higher number of vein ligations, and a higher number of artery preservations, according to our research. IVDU could be used in conjunction with subinguinal microsurgical varicocelectomy to improve surgical outcomes. 


\section{REFERENCES}

1. Juho YC, Wu ST, Kao CC, Meng E, Cha TL, Yu DS. Anatomic mapping of the internal spermatic vein via subinguinal varicocelectomy with intraoperative vascular Doppler ultrasound. J Chinese Med Assoc. 2019;82(2):115-9.

2. Clavijo RI, Carrasquillo R, Ramasamy R. Varicoceles: prevalence and pathogenesis in adult men. Fertil Steril. 2017;108(3):364-9.

3. Pagani RL, Ohlander SJ, Niederberger CS. Microsurgical varicocele ligation: surgical methodology and associated outcomes. Fertil Steril. 2019;111(3):415-9.

4. Hamada A, Esteves SC, Agarwal A. Definitions and Epidimiology. In: Varicocele and Male Infertility. Springer, Champ; 2016. p. 1-3.

5. Cocuzza M, Pagani R, Coelho R, Srougi M, Hallak J. The systematic use of intraoperative vascular Doppler ultrasound during microsurgical subinguinal varicocelectomy improves precise identification and preservation of testicular blood supply. Fertil Steril. 2010;93(7):2396-9.

6. Guo L, Sun W, Shao G, Song H, Ge N, Zhao S, et al. Outcomes of Microscopic Subinguinal Varicocelectomy With and Without the Assistance of Doppler Ultrasound: A Randomized Clinical Trial. Urology. 2015;86(5):922-8.

7. Lv KL, Zhang YD, Zhuang JT, Gao Y, Zhao L, Wan Z, et al. Subinguinal microsurgical varicocelectomy with intraoperative microvascular Doppler ultrasound leads to the pain-free outcome after surgery. J Xray Sci Technol. 2017;25(5):839-46.

8. Özkaptan O, Balaban M, Sevinc C, Çubuk A, Sahan A, Akca O. Impact of intra-operative doppler ultrasound assistance during microsurgical varicocelectomy on operative outcome and sperm parameters. Andrologia. 2020;52(7):e13641.

9. Chetan V R. Impact of intra-operative doppler ultrasound assistance during microsurgical varicocelectomy on operative outcome and sperm parameters. Int J Heal Clin Res. 2021;4(10):185188.

10. Güdeloğlu A, Karakurt G, Altan M, Ergen A. Micro-Doppler Ultrasonography-assisted Microsurgical Varicocelectomy: First Time in Turkiye. J Urol Surg. 2020;7(1):46-9.

11. Zini A. Technique Is the Treatment of Choice. Hum Reprod Update. 2007;1(3):273-6. 